\title{
Disruption of Conscious Access in Psychosis Is Associated with Altered Structural Brain Connectivity
}

\author{
Lucie Berkovitch, ${ }^{1,2}$ Lucie Charles, ${ }^{3}$ Antoine Del Cul, ${ }^{4}$ Nora Hamdani, ${ }^{5}$ Marine Delavest, ${ }^{6}$ Samuel Sarrazin, $, 2,7$ \\ Jean-François Mangin, ${ }^{2}$ Pamela Guevara, ${ }^{8}$ Ellen Ji, ${ }^{2,9}$ Marc-Antoine d'Albis, ${ }^{2,5,9}$ Raphaël Gaillard, ${ }^{1}$ \\ Frank Bellivier, ${ }^{6}$ Cyril Poupon, ${ }^{2}$ Marion Leboyer, ${ }^{5,9}$ Ryad Tamouza, ${ }^{5,9}$ Stanislas Dehaene, ${ }^{2,10}$ and \\ (ㄱ) Josselin Houenou ${ }^{2,5,9}$ \\ ${ }^{1}$ Groupe Hospitalier Universitaire Paris Psychiatrie et Neurosciences, Service Hospitalo-Universitaire Pôle Hospitalo-Universitaire \\ Psychiatrie Paris 15, 75014 Paris, France, ${ }^{2}$ Université Paris Saclay, CEA, Neurospin, F-91191, Gif-sur-Yvette, France, ${ }^{3}$ Institute of Cognitive \\ Neuroscience, UCL, London WC1E 6BT, United Kingdom, ${ }^{4}$ Service de Psychiatrie d'Adultes, Hôpital Universitaire Pitié Salpêtrière, 75013 \\ Paris, France, ${ }^{5}$ AP-HP, Hôpitaux Universitaires H. Mondor, DMU IMPACT, FHU ADAPT, F-94010, Créteil, France, ${ }^{6}$ Pôle neurosciences, Groupe \\ Hospitalier Saint-Louis, Lariboisière Fernand Widal Assistance Publique-Hôpitaux de Paris, 75010 Paris, France, ${ }^{7}$ Centre Médical \\ Pasteur, 94550 Chevilly-Larue, France, ${ }^{8}$ Faculty of Engineering, Universidad de Concepción, Concepción 4070386, Chile, ${ }^{9}$ Université Paris \\ Est Créteil, INSERM, IMRB, Translational Neuropsychiatry, Fondation FondaMental, F-94010, Créteil, France, and ${ }^{10}$ Collège de France, Paris \\ 75005, France
}

According to global neuronal workspace (GNW) theory, conscious access relies on long-distance cerebral connectivity to allow a global neuronal ignition coding for conscious content. In patients with schizophrenia and bipolar disorder, both alterations in cerebral connectivity and an increased threshold for conscious perception have been reported. The implications of abnormal structural connectivity for disrupted conscious access and the relationship between these two deficits and psychopathology remain unclear. The aim of this study was to determine the extent to which structural connectivity is correlated with consciousness threshold, particularly in psychosis. We used a visual masking paradigm to measure consciousness threshold, and diffusion MRI tractography to assess structural connectivity in 97 humans of either sex with varying degrees of psychosis: healthy control subjects $(n=46)$, schizophrenia patients $(n=25)$, and bipolar disorder patients with $(n=17)$ and without $(n=9)$ a history of psychosis. Patients with psychosis (schizophrenia and bipolar disorder with psychotic features) had an elevated masking threshold compared with control subjects and bipolar disorder patients without psychotic features. Masking threshold correlated negatively with the mean general fractional anisotropy of white matter tracts exclusively within the GNW network (inferior frontaloccipital fasciculus, cingulum, and corpus callosum). Mediation analysis demonstrated that alterations in longdistance connectivity were associated with an increased masking threshold, which in turn was linked to psychotic symptoms. Our findings support the hypothesis that long-distance structural connectivity within the GNW plays a crucial role in conscious access, and that conscious access may mediate the association between impaired structural connectivity and psychosis.

Key words: bipolar disorder; cerebral connectivity; consciousness; global neuronal workspace; schizophrenia; tractography

Received Apr. 17, 2020; revised Aug. 10, 2020; accepted Sep. 20, 2020.

Author contributions: A.D.C., M.L., R.T., S.D., and J.H. designed research; L.C., A.D.C., N.H., M.D., S.S., J.-F.M., P.G., E.J., M.A.d., F.B., C.P., M.L., and J.H. performed research; L.B., L.C., E.J., R.G., S.D., and J.H. analyzed data; L.B. wrote the paper.

This work was supported by the French-German ANR (Agence Nationale de la Recherche)/DFG (Deutsche Forschungsgemeinschaft) "FUNDO" Project; the French ANR under the "VIP" (MNP 2008) Project; the Investissements d'Avenir programs managed by the ANR under references ANR-11-IDEX-004-02 (Labex BioPsy) and ANR-10-COH0-10-01; Institut National de la Santé et de la Recherche Médicale; Commissariat à I'Energie Atomique; Collège de France; and Fondation pour la Recherche Médicale (Grants 40532 and DIC20161236445). The work was partially funded by the Human Brain Project, funded from the European Union's Horizon 2020 Framework Program for Research and Innovation under the Specific Grant Agreements
785907 (SGA2) and 604102 (SGA1). P. Guevara received funding from CONICYT FONDECYT 1190701, CONICYT PIA/Anillo de Investigacion en Ciencia y Tecnologia ACT172121 and CONICYT BASAL FB0008 grants. We thank all subjects for participation in this study. We also thank Philippe Le Corvoisier for assisting in the recruitment of healthy control subjects and the personnel of the UNIACT (Translational and Applicative Neuroimaging Research Unit) laboratory. In addition, we thank the editors and reviewers for insightful remarks and suggestions.

The authors declare no competing financial interests.

Correspondence should be addressed to Josselin Houenou at josselin.houenou@inserm.fr.

https://doi.org/10.1523/JNEUROSCI.0945-20.2020

Copyright $\odot 2021$ the authors 


\section{Significance Statement}

Although people with schizophrenia and bipolar disorder display cerebral dysconnectivity and impaired conscious access, the link between these two alterations and their involvement in psychopathology remains unclear. This study measured structural connectivity using diffusion MRI and consciousness threshold in healthy control subjects, schizophrenia patients, and bipolar disorder patients with and without psychotic features. The degree of deficits in conscious access was associated with psychosis: patients with psychosis (schizophrenia and bipolar disorder with psychotic features) demonstrated larger deficits in conscious access compared with control subjects and bipolar disorder patients without psychotic features. As predicted by the global neuronal workspace theory, cerebral connectivity within a specific network was negatively correlated with consciousness threshold. Finally, we found that conscious access impairments mediated dysconnectivity and psychosis.

\section{Introduction}

The global neuronal workspace (GNW) theory (Dehaene et al., 1998; Dehaene and Changeux, 2011) proposes that information becomes consciously accessible when it triggers sustained activity in a large network of interconnected neurons (Sergent et al., 2005; Del Cul et al., 2007; van Vugt et al., 2018), leading to the prediction that structural integrity within the posteroanterior long-distance network may be one of the prerequisites for conscious access. This proposal is supported by a study showing that consciousness threshold was correlated with structural connectivity in patients with multiple sclerosis (Reuter et al., 2009), although this hypothesis remains unexplored in other diseases showing structural dysconnectivity and impaired conscious access.

Patients with schizophrenia exhibit impairments in conscious access (Del Cul et al., 2006; Green et al., 2011; Mathis et al., 2012; Hanslmayr et al., 2013; Herzog et al., 2013; Berkovitch et al., 2018) and disorganized and/or insufficient myelinated fiber tracts (Klauser et al., 2017; Kelly et al., 2018), even before treatments (Li et al., 2018), particularly in the prefrontal cortex (Wu et al., 2015) and cingulum (Sun et al., 2003). Importantly, alterations in structural connectivity were shown to be associated with clinical features of the disease (Wolkin et al., 2003; Skelly et al., 2008; Canu et al., 2015; Kochunov et al., 2017; Griffa et al., 2019).

Dysconnectivity of white matter tracts has also been reported in patients with bipolar disorder (Magioncalda et al., 2016; Favre et al., 2019), especially those with psychotic features (Anticevic et al., 2013; Sarrazin et al., 2014), but to a lesser degree than that of patients with schizophrenia (Skudlarski et al., 2013). Only a few studies have explored their consciousness threshold, with mixed results (Fleming and Green, 1995; McClure, 1999; Goghari and Sponheim, 2008; Chkonia et al., 2012).

It has been proposed, but not yet tested, that a disruption in conscious access could increase the liability to psychosis because insufficient conscious access to information may cause cognitive impairments and make patients prone to developing false inferences that fuel delusional ideas (Berkovitch et al., 2017). The observation of shared dysconnectivity patterns between schizophrenia and bipolar disorder with psychotic features (McIntosh et al., 2008) also fits the hypothesis that psychotic symptoms may be related to an increased consciousness threshold.

The aim of this study was to test a three-step model in which structural dysconnectivity is correlated with an elevated consciousness threshold, which in turn is also associated with psychotic symptoms in patients. To do so, we measured structural connectivity with diffusion imaging-based tractography and the threshold for conscious access using a visual masking paradigm in participants with varying degrees of psychosis (control subjects, and patients with bipolar disorder and with schizophrenia).
The bipolar disorder patient group was subdivided according to the presence or absence of psychotic features, allowing us to examine the specificity of the abnormal consciousness threshold to psychosis. Generalized fractional anisotropy (gFA) was measured in predefined fiber bundles. The inferior fronto-occipital fasciculus (IFOF), cingulum long fibers (CLFs), and corpus callosum were chosen as a priori bundles of interest because they play a critical role in conscious access according to the GNW theory (Dehaene and Changeux, 2011; Hesselmann et al., 2013; Sarubbo et al., 2013; Forkel et al., 2014; Herbet et al., 2016). Inferior longitudinal fasciculi (ILFs) involved in early vision but not spatial neglect (Urbanski et al., 2008), and U-shaped short fibers that reflect the general integrity of white matter in the brain, were used as control subjects, to check whether the correlation between consciousness, as assessed by masking threshold, and cerebral connectivity was specific and restricted to GNW fibers (Fig. 1, right).

Following the predictions of the GNW theory (Dehaene et al., 1998; Dehaene and Changeux, 2011), we posited that long-range cerebral connectivity would correlate with masking threshold in the general population, including patients. We further predicted that patients with schizophrenia, and to a lesser extent patients with bipolar disorder, would exhibit an elevated masking threshold that would be associated with psychotic features.

\section{Materials and Methods}

\section{Participants}

We analyzed data from 97 humans of either sex (51 patients, 46 control subjects). Among patients, there were 26 patients with bipolar disorder type 1 or 2 ( 9 without psychotic features and 17 with psychotic features) and 25 patients with schizophrenia or schizoaffective disorder, according to DSM-IV (Diagnostic and Statistical Manual of Mental Disorders, 4th edition) criteria. They were recruited from two psychiatry departments of university-affiliated hospitals [APHP (Assistance Publique-Hôpitaux de Paris), Mondor University Hospitals, Créteil and Fernand Widal Lariboisière, Paris, France] and were mostly outpatients. History of psychotic features was defined as having at least one previous manic or depressive episode with delusions or hallucinations. The initial sample size was 99 participants, but 1 patient with bipolar disorder without psychotic features and 1 patient with schizophrenia were excluded because their masking thresholds were abnormally high (see Results). Control participants were free of any past or present psychiatric disorder and first-degree family history of bipolar disorder, schizophrenia, or schizoaffective disorder. The inclusion criteria for all participants were as follows: between 18 and 60 years of age, no history of alcohol or drug abuse/dependence, no previous head trauma with a loss of consciousness, no current or past cardiac or neurologic disease, and no contraindications for MRI.

The study was approved by the ethical committee of CPP Mondor University Hospital (Créteil, France). Written informed consent was obtained for all subjects before their participation in the study. We 


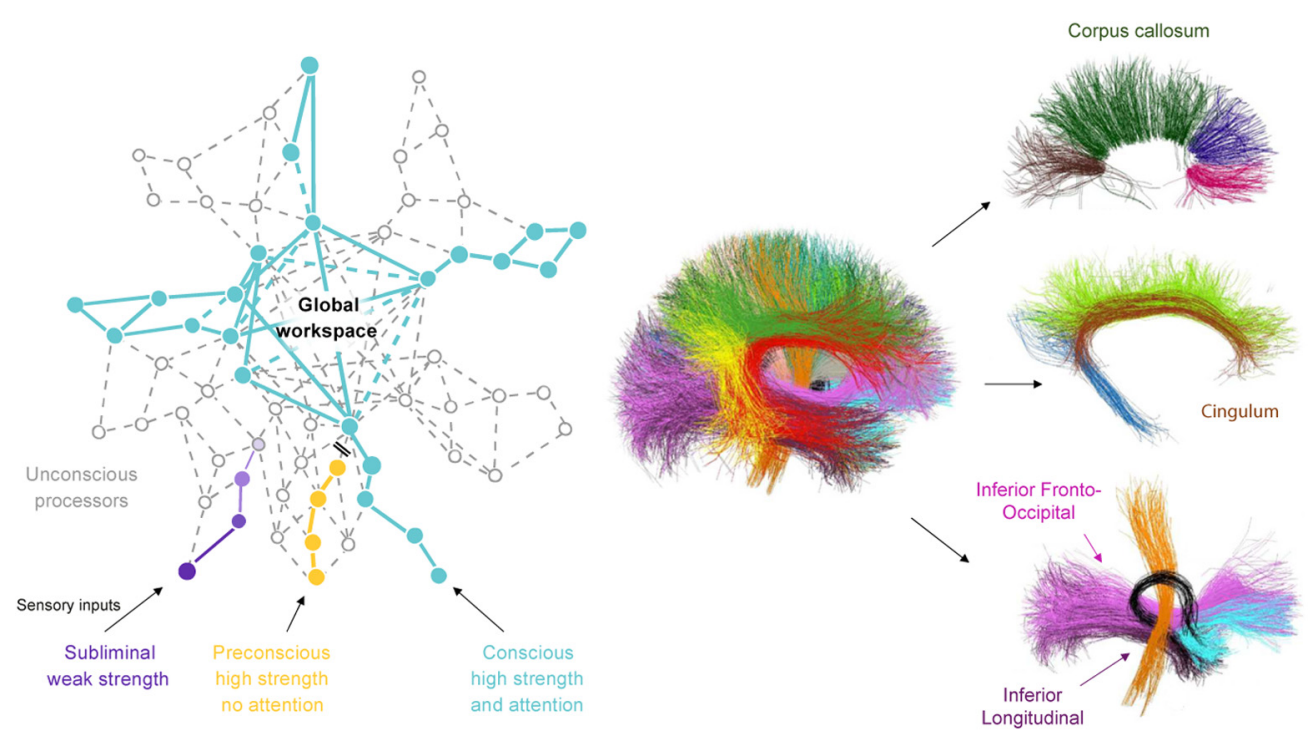

Figure 1. Predictions of the global neuronal workspace and detailed view of the bundles of interest. Left, The global neuronal workspace theory assumes that information becomes consciously accessible when it is amplified by attention and triggers sustained activity in a large network of interconnected neurons. The long-distance connectivity of higher cortical areas, particularly prefrontal cortex, therefore plays an essential role in conscious access. Right, We conducted an imaging analysis on bundles supposedly involved in the global workspace. IFOFs (pink) and CLFs (brown) correspond to long-distance posteroanterior connections, and the corpus callosum that underlies interhemispheric communication and the formation of a single bihemispheric state of ignition. Additional bundles were included in the analysis as control subjects (occipitotemporal ILFs, purple; U-shaped short fibers, not represented in this figure) to check whether a correlation between masking threshold and cerebral connectivity was specific to fibers involved in the global neuronal workspace.

calculated olanzapine equivalents following international expert consensus-based recommendations (Leucht et al., 2016). Treatment information was missing for two patients with bipolar disorder. Current psychotic symptoms were rated using the Positive and Negative Symptom Scale (PANSS; Kay et al., 1987). We also examined the effect of other parameters such as age of disease onset, total number of manic-depressive and psychotic episodes, number of hospitalizations, and tobacco and cannabis use. Participants' characteristics and missing data are reported in Table 1.

\section{Experimental design}

Consciousness threshold measure. Stimuli and procedures were identical to those in the study by Del Cul et al. (2009), using a variant of the masking paradigm adapted for normal and clinical populations (Del Cul et al., 2006, 2007; Reuter et al., 2007, 2009; Charles et al., 2017; Berkovitch et al., 2018; Fig. 2). A numerical target digit (0-9) was presented for a fixed duration of $\sim 17 \mathrm{~ms}$ at a randomly chosen position among four possible positions. After a variable delay [stimulus onset asynchrony (SOA)], a mask appeared at the target location for $250 \mathrm{~ms}$. The mask was composed of four letters surrounding the original target stimulus location without superimposing or touching it (metacontrast masking). For each trial, subjects were asked to rate the visibility of the target (seen or unseen, subjective measure of consciousness) and then name the masked digit under forced-choice instructions (objective measure of consciousness). Target-mask SOA varied on a trial-by-trial basis using a "double staircase" algorithm (Del Cul et al., 2009) to determine the masking threshold. Each trial was randomly assigned to one of the two staircases, one starting with the shortest SOA $(17 \mathrm{~ms})$, and the other with the longest SOA $(133 \mathrm{~ms})$. Independently for each staircase, the stimulus-mask SOA was decreased by one frame of the computer screen ( $17 \mathrm{~ms}$ at a $60 \mathrm{~Hz}$ refresh rate) whenever the digit was consciously perceived (i.e., when the subject simultaneously reported seeing the stimulus on the previous trial and was correct in identifying the digit, such that the digit would be more difficult to consciously perceive at the subsequent trial). Otherwise, if the participant did not report that they saw the stimulus on the previous trial or incorrectly identified the digit, the digit was considered as being under the consciousness threshold and the SOA was increased by one frame to make the digit easier to perceive. The masking threshold, that supposedly reflects the consciousness threshold, corresponded to the mean SOA, averaged over the 18 most recent alternations between an increase and a decrease.

MRI acquisition. All participants were scanned at NeuroSpin neuroimaging center on the 3 T Magnetom TrioTim syngo MR B17 with a 12channel head coil (Siemens Medical Solutions). The MRI protocol included a high-resolution T1-weighted acquisition (TE, $2.98 \mathrm{~ms}$; TR, $2300 \mathrm{~ms}$; 160 sections; voxel size, $1.0 \times 1.0 \times 1.1 \mathrm{~mm})$, a diffusionweighted (DW) sequence along 60 directions (voxel size, $2.0 \times 2.0 \times 2.0$ $\mathrm{mm}$; $\mathrm{b}$-value $=1400 \mathrm{~s} / \mathrm{mm}^{2}$ plus 1 image in which $\mathrm{b}=0$; TE, $92 \mathrm{~ms}$; TR, $12 \mathrm{~s} ; 60$ axial sections) and a field map. Scans were acquired on the same day as the psychiatric evaluation and the behavioral measure of consciousness threshold (immediately before or after the scanning session). Each participant's data were assessed for movement, susceptibility, and noise artifacts by researchers who were blinded to the diagnosis.

$D W$ image data processing. We used a processing pipeline of DW images (DWIs) similar to those used in previous studies (Sarrazin et al., 2014; Souza-Queiroz et al., 2016). DW MRI data were processed with Connectomist 2.0 and BrainVisa 4.2 software (http://www.brainvisa. info). The DW images were corrected for noise/spikes with q-space interpolation correction and for susceptibility artifacts using the field map. We then computed an orientation distribution function at each voxel included in this mask using an analytical QBI (Q-ball imaging) model (spherical harmonic order, 6; regularization factor, $\lambda=0.006$; Descoteaux et al., 2007). We evaluated the gFA from all the computed orientation distribution functions (Tuch, 2004). A decreased gFA value is thought to indicate the loss of integrity or loss of coherence of white matter (Le Bihan and Johansen-Berg, 2012).

We used a T1-based propagation tractography mask (Guevara et al., 2012) and performed whole-brain tractography in each subject's native space using a regularized streamline deterministic algorithm (one seed per voxel; forward step, $0.5 \mathrm{~mm}$; bilateral propagation). Algorithm propagation was interrupted if the tract length exceeded $300 \mathrm{~mm}$, if the tract streamline propagated outside the mask, or if the curvature between two steps exceeded $30^{\circ}$. No between-subject registration was performed.

Whole-brain tractography volumes were then segmented using an automatic segmentation pipeline based on a clustering technique relying on the definition of a pairwise distance between fibers and multisubject bundle atlases, described in depth previously (Guevara et al., 2012). This process leads to the segmentation of the selected bundles from subject tractography datasets (i.e., 22 known deep white matter bundles, and U- 
Table 1. Participants' characteristics

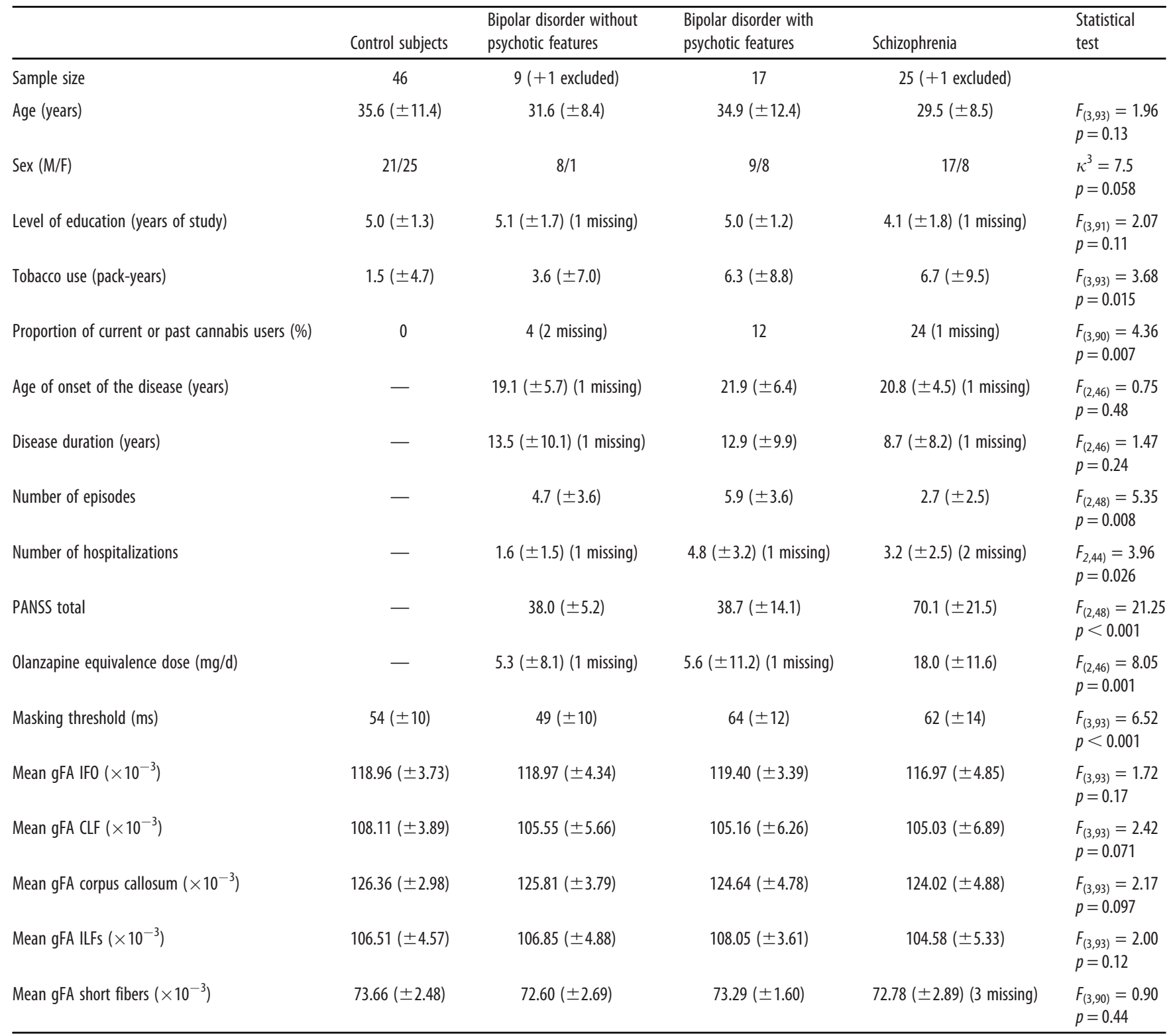

Values are given as the mean $( \pm S D)$. F, Female; $M$, male.

fiber bundles; Guevara et al., 2017; Román et al., 2017), allowing a comprehensive whole-brain exploration of white matter connectivity. We then extracted the mean gFA along the bundles for each subject using BrainVISA software.

We conducted analyses on predefined fiber bundles that were chosen based on their critical involvement in conscious access according to the GNW theory (Dehaene and Changeux, 2011). We averaged the mean gFA of left and right bundles for IFOFs, CLFs, and ILFs of the four subparts of the corpus callosum (i.e., genu, body, splenium, and rostrum) and aggregated all the short fibers to have an overall estimate of the gFA. Data for short fibers were missing for three participants.

\section{Statistical analysis}

We used a correlational approach to estimate whether the threshold for conscious access was linked to clinical features and cerebral connectivity. Welch's two-sample $t$ tests, ANOVAs, and Pearson's correlation were conducted on a masking threshold, with clinical and imaging characteristics as within-subject factors. Regarding clinical characteristics, factors included in ANOVAs were diagnosis (bipolar disorder with and without psychotic features, schizophrenia vs control subjects), psychosis (presence in schizophrenia and bipolar disorder with psychotic features vs absence in control subjects and bipolar disorder without psychotic features), olanzapine equivalent daily doses, PANSS total scores, age of onset, disease duration, number of episodes, number of hospitalizations, and tobacco and cannabis use. ANOVAs were run on masking threshold values with mean gFA and clinical characteristics as within-subject factors. Pearson correlations between masking threshold and mean gFA were conducted separately for each bundle. Whenever mentioned, statistical results were adjusted for multiple comparisons using the Bonferroni method (p.adjust function in $\mathrm{R}$ software). Effect sizes were estimated using Cohen's d (d.cohen function in R software).

Because some of the hypotheses at stake lie on an absence of difference (e.g., no correlation between masking threshold and mean gFA of bundles that do not belong to the GNW), in addition to frequentist statistics [values of the statistic (e.g., $t$ or $F$ values), as well as $p$ values are reported], we also conducted Bayesian statistics whenever required. Contrary to frequentist statistics, Bayesian statistics symmetrically quantify the evidence in favor of the null $\left(\mathrm{H}_{0}\right)$ and the alternative $\left(\mathrm{H}_{1}\right)$ hypotheses, therefore allowing the conclusion in favor of an absence of difference (Wagenmakers et al., 2010). 


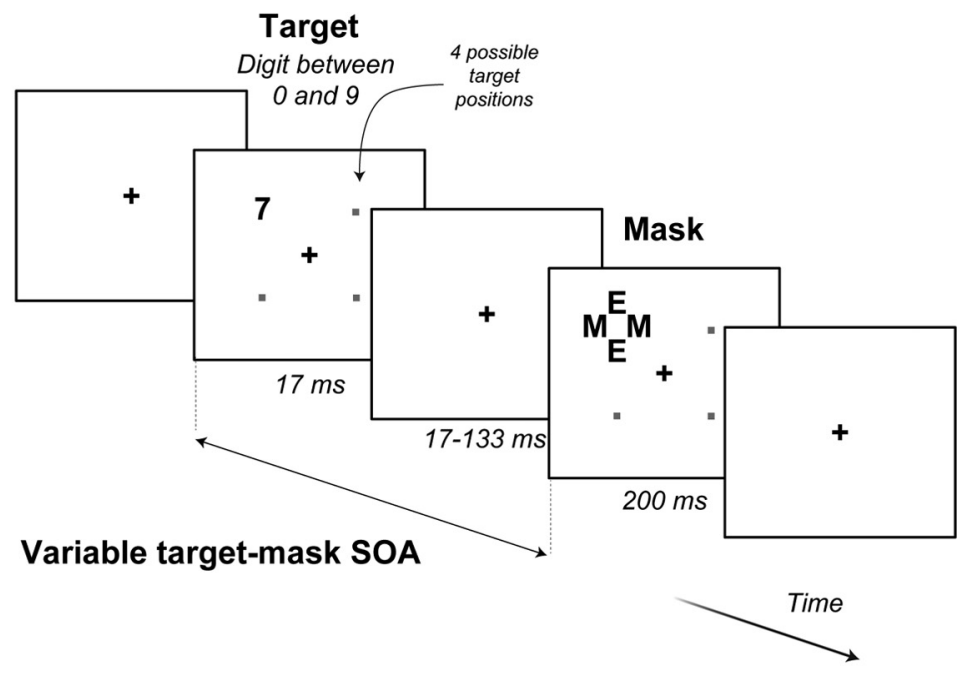

Questions

Double staircase algorithm

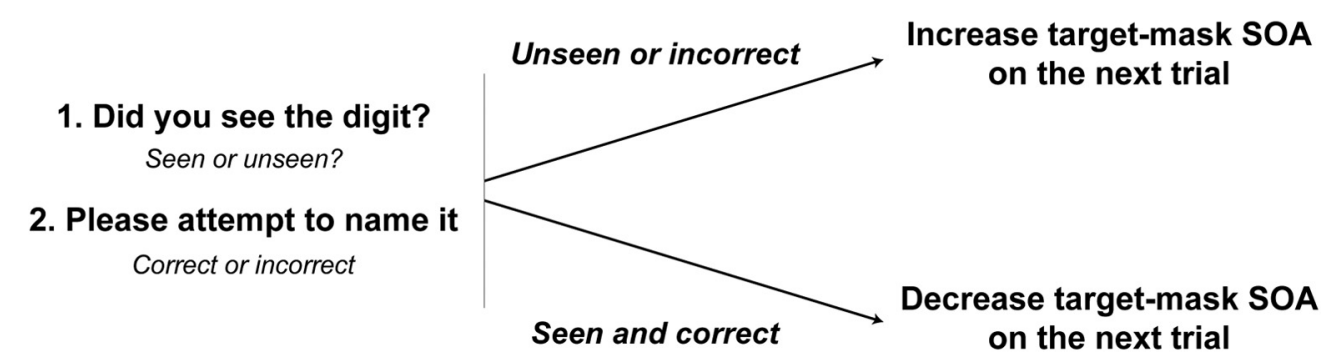

Figure 2. Experimental paradigm. To determine the consciousness threshold, a double staircase algorithm was used. A digit target was presented for $17 \mathrm{~ms}$ and masked after a variable delay (SOA) by a metacontrast mask composed of four letters. Participants indicated whether they saw the digit or not and were asked to identify the digit presented. If the target was both seen and correctly named, the target-mask SOA was decreased on the next trial, making the target more difficult to consciously perceive. Otherwise (unseen and/or incorrect answers), the target-mask SOA was increased.

To do so, we used the BayesFactor package (http://bayesfactorpcl.r-forge.rproject.org) implemented in $\mathrm{R}$ (https://www.r-project.org). Bayes factors (BFs) were estimated using a scale factor of $r=0.707$. For each Bayesian statistical test, we reported the corresponding Bayes factor $\left(\mathrm{BF}_{10}=p\left(\right.\right.$ data $\left.\mid \mathrm{H}_{1}\right) /$ $p\left(\right.$ data $\left.\left.\mid \mathrm{H}_{0}\right)\right)$. Although threshold values of BFs have been proposed (e.g., a $\mathrm{BF}>3$ is usually taken as providing substantial evidence), a BF value of $x$ can directly be interpreted as the observed data being approximately $x$ times more probable under the alternative compared with the null hypothesis. When BFs favored the null hypotheses (i.e., $\mathrm{BF}_{10}<1$ ), we directly reported the inverse Bayes factor (i.e., $\mathrm{BF}_{01}=1 / \mathrm{BF}_{10}$ ) quantifying the evidence in favor of the null compared with the alternative hypothesis.

Finally, we examined the link among connectivity, masking threshold, and psychotic symptoms across subjects, using a mediation analysis (Baron and Kenny, 1986; but see Shrout and Bolger, 2002). We hypothesized that dysconnectivity would elevate the consciousness threshold, which would in turn increase psychotic symptoms. Mean gFA was explored as a predictor variable of masking threshold with a first linear model. Then, in a second linear model, the presence of psychotic features was included as a binary outcome variable ( 0 for control subjects and patients with bipolar disorder without psychotic features, 1 for patients with bipolar disorder with psychotic features and patients with schizophrenia), explained by mean gFA (predictor variable) and masking threshold (mediator variable). The two linear models were entered in a mediation analysis with 10,000 simulations, using the mediate function included in the $\mathrm{R}$ software mediation package (Tingley et al., 2014; https://www.r-project.org). The mediator variable (consciousness threshold) was explored as a mediator that explains the underlying relationship between the predictor variable (cerebral connectivity) and the outcome variable (psychotic symptoms). Direct effects reflect the influence of the predictor variable on the outcome that is not mediated by the mediator variable (i.e., the direct effect of dysconnectivity on the advent of psychotic features) and mediation effects reflect the influence of the predictor variable on the outcome that is mediated by the mediator variable. If the mediation effect is significant and direct effects completely disappear when performing mediation analysis, it means that the effect of the predictor variable on the outcome variable is fully mediated by the mediator variable. If both direct and mediation effects are significant, it means that the effect is partially mediated by the mediator variable.

Results are expressed as $p$ values (significant at $<0.05$ ), Welch's $t$ value, Pearson's $r$ value, $F$ value, Cohen's $d$, Bayes factors or their inverse, and quasi-Bayesian 95\% confidence intervals.

\section{Results}

Behavioral results: masking threshold is increased in patients with psychotic features

We first examined whether the masking threshold varied with clinical characteristics. Two participants (one patient with bipolar disorder, one with schizophrenia) were excluded because their masking thresholds were $>3$ SDs above the group mean (134 and $97 \mathrm{~ms}$, respectively), resulting in a final sample of 97 participants. Behavioral data and participants' characteristics are summarized in Table 1 and behavioral results are shown in Figure 3. ANOVA on masking threshold with diagnosis as 


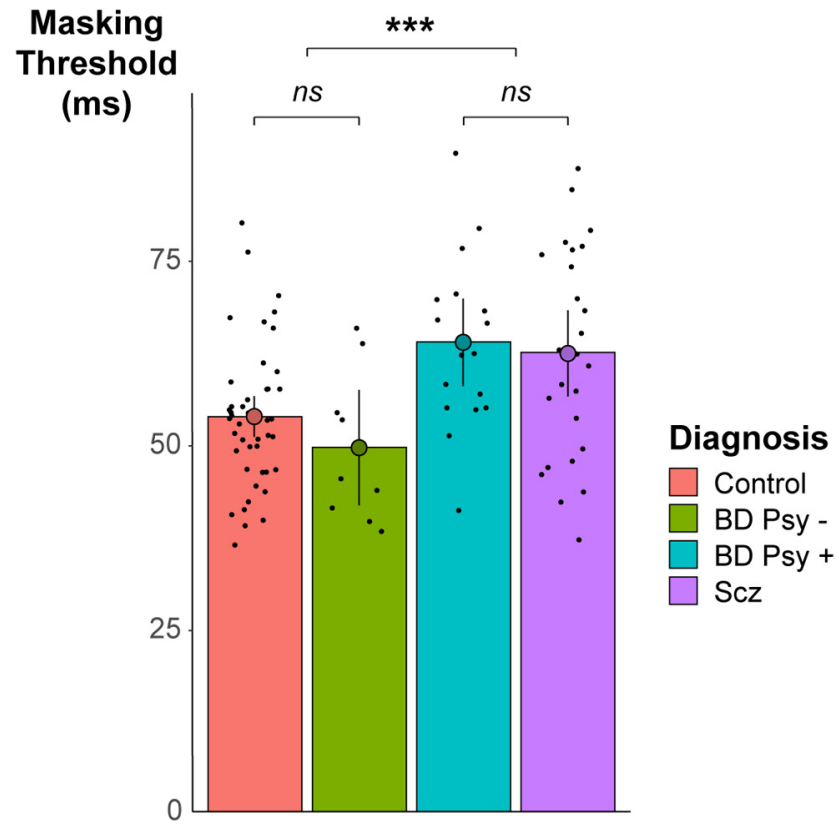

Figure 3. Behavioral results. The masking threshold was significantly increased in patients with psychosis (i.e., with bipolar disorder associated with psychotic features (BD Psy +; blue) and with schizophrenia (Scz; purple) compared with control subjects (pink) and patients with bipolar disorder without psychotic features (BD Psy -; green). $* * * p<0.001$. ns, Nonsignificant. Bars represent $95 \%$ confidence intervals.

within-subject factor revealed a significant effect of diagnosis on masking threshold $\left(F_{(3,93)}=6.52, p<0.001\right)$. We therefore performed five post hoc $t$ tests to further explore the diagnosis effect on masking threshold; $p_{\text {adjusted }}$ corresponds to $p$ values after Bonferroni adjustments, correcting for the five performed $t$ tests. A significant difference in threshold was observed between participants with and without psychosis (i.e., patients with schizophrenia and patients with bipolar disorder and psychotic features vs control subjects and patients with bipolar disorder without psychotic features; 53 vs $63 \mathrm{~ms}, t_{(72.1)}=-4.14, p<0.001$, $p_{\text {adjusted }}<0.001$, Cohen's $\left.d=-1.27\right)$. This difference was also observable within the bipolar disorder patients (without psychotic symptoms, $49 \mathrm{~ms}$; vs with psychotic symptoms, $63 \mathrm{~ms}$; $t_{(18.36)}=-3.20, p=0.005, p_{\text {adjusted }}=0.024$, Cohen's $\left.d=-0.88\right)$. Bipolar disorder patients without psychotic features did not differ from control subjects regarding their masking threshold ( $49 \mathrm{~ms}$ versus $54 \mathrm{~ms}, t_{(10.8)}=1.11, p=0.29, \mathrm{BF}=1 / 1.72$ ). Bipolar disorder patients with psychotic features did not differ from patients with schizophrenia $\left(t_{(38.6)}=-0.35, p=0.73, \mathrm{BF}=1 / 3.11\right)$ and had a significantly higher masking threshold than healthy control subjects ( 64 vs $54 \mathrm{~ms}, t_{(24.2)}=-3.21, p=0.004$, $p_{\text {adjusted }}=$ 0.019, Cohen's $d=1.00)$.

Among patients, there was no significant association between olanzapine equivalent daily doses and the masking threshold (Pearson $r=0.25, t_{(47)}=1.80, p=0.079, \mathrm{BF}=1.29$ ), while PANSS total scores were significantly and positively correlated with threshold $\left(r=0.28, t_{(49)}=2.06, p=0.045\right)$. There was no significant effect of age at disease onset, number of manic-depressive or psychotic episodes or cannabis use on threshold (all $p>0.2$ ). By contrast, the number of hospitalizations, the duration of disease, and tobacco use were significantly and positively correlated to the masking threshold (number of hospitalizations: $r=0.30$, $t_{(45)}=2.04, p=0.047$; duration of disease: $r=0.36, t_{(47)}=2.68$, $p=0.010$; tobacco use: $r=0.30, t_{(49)}=2.18, p=0.035$ ).
Anatomical connectivity correlates with masking threshold Across subjects (patients and control subjects), the masking threshold was significantly and negatively correlated with the mean gFA of the IFOF (Pearson $r=-0.28, t_{(95)}=-2.84$, $p=0.006, \mathrm{BF}=8.87)$, CLFs $\left(r=-0.27, t_{(95)}=-2.75, p=0.007\right.$, $\mathrm{BF}=7.17)$, and corpus callosum $\left(r=-0.30, t_{(95)}=-3.09\right.$, $p=0.003, \mathrm{BF}=17.1$; Fig. 4 , top). Note that a negative correlation implies that a greater anisotropy is associated with a lower threshold and therefore an improved conscious perception, as predicted by the GNW hypothesis. These correlations remained significant after Bonferroni adjustments, correcting for the three bundles tested (IFOF: $p_{\text {adjusted }}=0.018$; CLFs: $p_{\text {adjusted }}=0.021$; corpus callosum: $p_{\text {adjusted }}=0.009$ ).

Crucially, for control bundles (ILFs and short fibers), mean gFA did not significantly correlate with masking threshold (ILFs: $r=-0.17, t_{(95)}=-1.63, p=0.1, \mathrm{BF}=1 / 1.25$; short fibers: $r=$ $\left.-0.09, t_{(92)}=-0.82, p=0.41, \mathrm{BF}=1 / 3.08\right)$.

To further confirm that the mean gFA of bundles belonging to the GNW had a significantly stronger correlation to the masking threshold than the mean gFA of bundles that are not involved in the GNW, we entered the gFA in an ANOVA with (1) a binary variable reflecting whether or not the bundle is involved in the GNW, (2) masking threshold as a within-subjects factor, and (3) subject as a random factor. This analysis showed a significant interaction between bundle role in GNW and threshold $\left(F_{(1,95)}=6.66\right.$, $p=0.011$ ), confirming our initial finding that the correlation between masking threshold and mean gFA was significantly higher for bundles belonging to the GNW than for control bundles.

We then checked interactions between mean gFA and participants' characteristics (diagnosis, psychotic features, medication, PANSS total, tobacco and cannabis use, age at onset, duration of the disease, number of episodes, number of hospitalizations) on masking threshold. Because many of these variables were interrelated, we performed separate ANOVAs on masking threshold for each variable, with mean gFA and the variable in question as within-subject factors. First, the effect of mean gFA remained significant when including the above factors in the analysis (all $F>4$, all $p<0.05$ ). Correlations between masking threshold and mean gFA did not significantly differ according to diagnosis, PANSS total, and tobacco use (all $F<1.5$; all $p>0.2$ ), but the number of hospitalizations, the duration of the disease, and the presence of psychotic features significantly interacted with mean gFA for some or all bundles $\left(\mathrm{gFA} \times\right.$ number of hospitalizations: IFOF: $F_{(1,43)}=4.35, p=0.043$; CLFs: $F_{(1,43)}=4.89, p=0.032$; and corpus callosum: $F_{(1,43)}=8.01$, $p=0.007$; gFA $\times$ duration of the disease for corpus callosum: $F_{(1,45)}$ $=8.43, p=0.006$; other bundles: all $F_{(1,45)}<1.5$, all $p>0.2$; gFA $\times$ psychotic features for CLFs: $F_{(1,93)}=3.95, p=0.049$; other bundles: all $F_{(1,93)}<1.5$, all $p>0.2$ ). In addition, structural connectivity was significantly reduced in patients with psychosis for CLFs and corpus callosum (effect of psychotic features on mean gFA for CLFs: $F_{(1,95)}$ $=5.59, p=0.020$; corpus callosum: $F_{(1,95)}=6.23, p=0.014$; but IFOF: $\left.F_{(1,95)}=1.46, p=0.23\right)$.

Finally, we used mediation analysis to explore the link among connectivity, masking threshold, and psychotic symptoms. We hypothesized that dysconnectivity would be associated with an elevated consciousness threshold, which would in turn be associated with psychotic symptoms. We first checked with a linear model whether mean gFA significantly influenced the presence of psychotic symptoms and found that it was the case for the CLFs and the corpus callosum but not for the IFOF (CLFs: $t=$ $-2.36 ; p=0.020$; corpus callosum: $t=-2.50, p=0.014$; IFOF: $t=$ $-1.21, p=0.22$ ). We already found that masking threshold was significantly correlated with mean gFA for the three bundles (see 

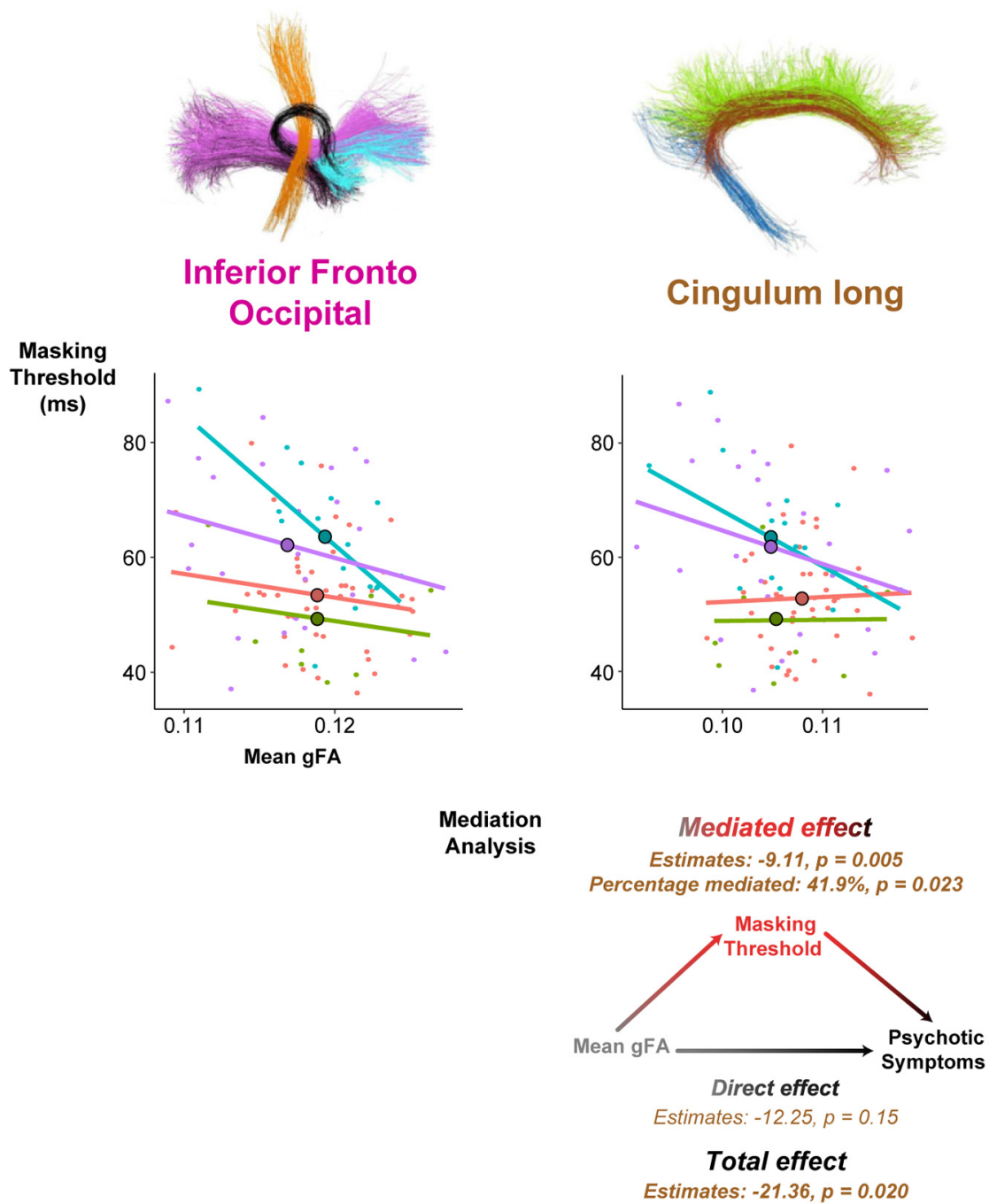

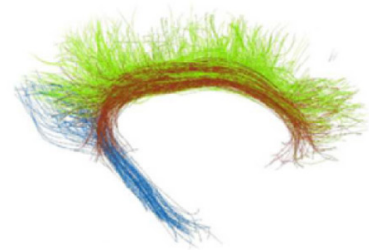

Cingulum long
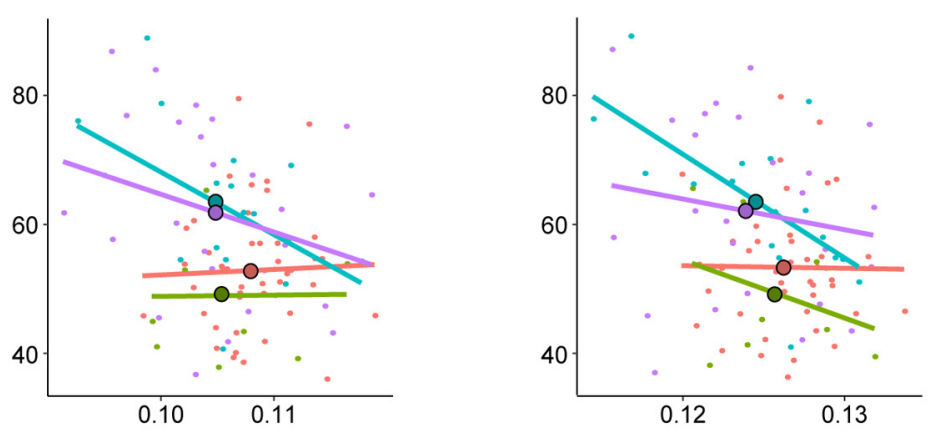

Corpus Callosum

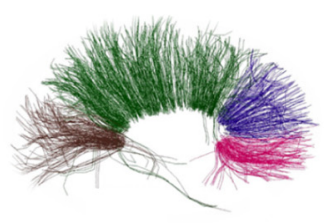
Controls BD PBD P+ Scz

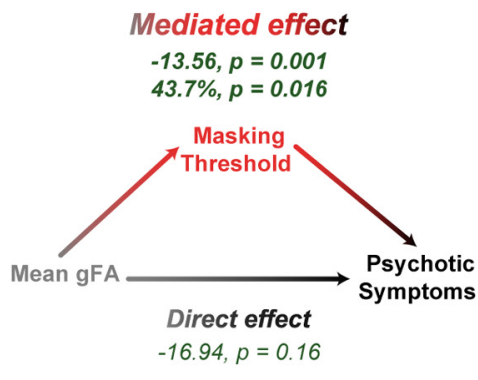

Total effect

$-30.70, p=0.014$

Figure 4. Correlation between masking threshold and the mean of gFA and mediation analysis for the three bundles of interest. Top, Masking threshold is negatively correlated with mean gFA across subjects for the inferior frontal-occipital fasciculus, the cingulum, and the corpus callosum, suggesting that conscious access relies on structural cerebral connectivity of these bundles. The masking threshold is represented as a function of the mean of gFA in each subgroup of participants. Each participant is represented in the point cloud (pink, control subjects; green, patients with bipolar disorder without psychotic features; patients with bipolar disorder and psychotic features (BD Psy-), blue; patients with schizophrenia (Scz; BD Psy +), purple. Mean of the masking threshold and the mean gFA in each group is represented by the dots with black outlines on the regression lines. Bottom, Mediation analysis with estimates and $p$ values of mediated, direct, and total effects for CLFs and corpus callosum. Mean gFA of the CLFs and the corpus callosum significantly influenced the presence of psychotic symptoms but not that of the IF0. Mediated effects were significant, while direct effects were not, which means that the effect of cerebral connectivity on the advent of psychotic symptoms was fully mediated by the masking threshold.

above). The effects of mean gFA on psychotic symptoms were no more significant when masking threshold was added in the linear model as a covariable (CLFs: $t=-1.40, p=0.17$; corpus callosum: $t=-1.41, p=0.16$ ), suggesting that the effect of mean gFA on psychotic symptoms was mediated by masking threshold. We thus performed mediation analysis for CLFs and corpus callosum. Results indicated that the correlation between decreased mean gFA and psychotic features was mediated by elevated masking threshold for these bundles (CLFs: ACME (Average Causal Mediation Effects): $\mathrm{CI}=-18.35$ to -2.17$], p=0.005$; corpus callosum: ACME: $\mathrm{CI}=-26.76$ to $-3.79, p=0.001)$. No direct effect yielded a significant result (all $p>0.1$ ). Those results suggest that a reduced gFA does not induce psychotic symptoms directly, but exclusively through its effect on the consciousness threshold (Fig. 4, bottom).

\section{Discussion}

Using a visual backward masking paradigm, we estimated the threshold for conscious access in patients with schizophrenia and bipolar disorder, and in control subjects, and explored whether it was correlated to structural connectivity, assessed by diffusion imaging-based tractography. In accordance with the predictions of the GNW theory (Dehaene and Changeux, 2011), we found an association between masking threshold and long-range structural connectivity in the IFOF, CLFs, and corpus callosum across subjects. Moreover, patients with psychosis (schizophrenia and bipolar disorder with psychotic features) exhibited an elevated masking threshold compared with control subjects and patients without psychosis. Finally, we conducted a mediation analysis suggesting that the link between alterations in connectivity and psychotic symptoms was mediated by an increased masking threshold, a result that fits with our previous proposals (Berkovitch et al., 2017).

The GNW theory assumes that conscious perception arises from the stabilization of neuronal activity within a distributed network, rendering a specific piece of information reportable to others (Dehaene et al., 1998; Dehaene and Changeux, 2011). In our study, we distinguished fiber bundles playing a critical role 
in the GNW (IFOF, CLFs, and corpus callosum) from control bundles (ILFs and U-shaped short fibers) in regard to their association with consciousness threshold. Specifically, we found a correlation between mean gFA and masking threshold for IFOF, CLFs, and corpus callosum across participants but not for the ILFs and the short U-shaped fibers. This result strongly supports the idea that conscious access relies on a specific long-distance network. This distinction between bundles inside and outside the GNW could be generalized to the whole brain to further test the properties of the GNW.

The clinical populations included in this study are known to exhibit dysconnectivity in GNW bundles (Pettersson-Yeo et al., 2011; Sarrazin et al., 2014); therefore, we expected their consciousness threshold to be increased. Our behavioral data confirmed this hypothesis and reproduced previous findings showing that patients with schizophrenia have an elevated consciousness threshold in backward masking (Butler et al., 2003; Del Cul et al., 2006; Green et al., 2011; Herzog et al., 2013; Berkovitch et al., 2018), inattentional blindness (Hanslmayr et al., 2013), and attentional blink (Mathis et al., 2012) paradigms (for review, see Berkovitch et al., 2017). Furthermore, we observed distinct profiles for patients with bipolar disorder according to the presence or absence of psychotic features: only patients with psychotic features had an elevated masking threshold. Such findings may account for the contrasted results previously obtained in this population (Fleming and Green, 1995; McClure, 1999; Goghari and Sponheim, 2008; Chkonia et al., 2012). In our study, patients exhibited an elevation of consciousness threshold that correlated with the PANSS, but they were mostly outside of acute episodes. In line with previous findings (Fleming and Green, 1995; Green et al., 1999), this suggests that conscious access alterations could constitute a symptomatic dimension (Allardyce et al., 2007; Henry and Etain, 2010) and may be a trait marker (Saccuzzo and Braff, 1986) to both schizophrenia and bipolar disorder with psychotic features.

Information sharing within the GNW during conscious perception has already been explored through functional connectivity during resting-state or task-based paradigms (Sergent et al., 2005; Del Cul et al., 2007; Barttfeld et al., 2015; van Vugt et al., 2018; Demertzi et al., 2019). In our study, we focused on anatomic structural connectivity as a preliminary step to understand the links between structural cerebral connectivity and conscious access in control subjects and patients. Several studies suggested that structural and functional connectivity are correlated, particularly within the default-mode network (Honey et al., 2007, 2009; Greicius et al., 2009; Horn et al., 2014) even if dissociations between structural and functional connectivity also exist, for instance during anesthesia (Boly et al., 2012; Lee et al., 2013; Schroeder et al., 2016). In psychiatric conditions, functional connectivity varies according to the clinical phase of the disease (Higashima et al., 2007; Magioncalda et al., 2015; Martino et al., 2016a), for instance between manic and depressive states in bipolar disorder (Magioncalda et al., 2015; Martino et al., 2016a). In those patients, alterations in functional and structural connectivity were shown to be correlated with different symptoms (Martino et al., 2016b) and acute state-dependent structural connectivity could change during a mood episode (Magioncalda et al., 2016). Additional valuable results may be obtained by exploring information sharing within the GNW using fMRI or EEG (King et al., 2013; Barttfeld et al., 2015) in patients, during and outside acute episodes, and understood in light of other consciousness theories such as the temporospatial theory of consciousness (Northoff and Huang, 2017; Northoff et al., 2020).
The specific elevation of masking threshold in patients with psychosis and its interaction with structural connectivity suggests a connection among psychosis, consciousness, and cerebral connectivity. We previously proposed that altered conscious access could favor the advent of psychotic symptoms (Berkovitch et al., 2017). Our results, including the mediation analysis, now allow us to propose a three-step model of psychosis in which dysconnectivity leads to a disruption of conscious access that ultimately translates into psychotic symptoms. Interestingly, our results are compatible with previous data showing a dissociation between preserved subliminal and altered conscious processing in schizophrenia (Höschel and Irle, 2001; Dehaene et al., 2003; Huddy et al., 2009; Grandgenevre et al., 2015; Charles et al., 2017), since subliminal processing is supposed not to involve long-distance connectivity. The increased difficulty for unconsciously processed information to reach consciousness may give rise to psychotic symptoms through several routes. An elevated consciousness threshold may significantly decrease the amount of information entering consciousness, promoting fictive interpretations, and overweighing the few sensory contents bursting into consciousness. Moreover, preserved unconscious processing may continue to implicitly guide behavior and fuel intuitions that cannot be consciously explained, fostering delusional constructions disconnected from the external world (Berkovitch et al., 2017).

An extensive literature on hierarchical predictive-coding brain mechanisms proposed that hallucinations and delusions could respectively result from an imbalance in the combination between prior knowledge and sensory inputs, and a failure to update beliefs according to incoming prediction error signals (Fletcher and Frith, 2009; Adams et al., 2013; Powers et al., 2017). Because of conflicting experimental results, it was recently proposed that computations between prior knowledge and sensory inputs at lower levels of the inference hierarchy may not be linearly related to those at higher levels (Sterzer et al., 2018; Corlett et al., 2019). Consciousness threshold thereby offers a plausible boundary between "low" (nonconscious) and "high" (conscious) levels, ruled by distinct Bayesian computations, crucially differently affected in schizophrenia. Interestingly, the model of circular inferences proposed by Jardri et al. (2017) provides a computational account of the relative overweight of the little sensory evidence crossing the consciousness threshold that could be reverberated in the GNW.

The causal role of impaired conscious access for psychotic features seems at odds with previous data that found a link between dysconnectivity and elevated consciousness threshold in pathologic conditions with no psychotic symptoms (Del Cul et al., 2009; Reuter et al., 2009). A possible explanation is that some but not all kinds of impairments in connectivity and conscious perception translate into psychotic symptoms. In particular, psychotic symptoms may only appear when dysconnectivity results from a particular neurophysiological dysfunction or is associated with other impairments. In this regard, results obtained through drug studies on the effects of ketamine, NMDA receptor antagonist that induces reversible psychotic symptoms at low doses and anesthesia at high doses, could help to provide a putative mechanism, at a molecular level, for linking dysconnectivity, elevated consciousness threshold, and psychotic symptoms. Indeed, ketamine anesthetic effects involve a disruption of long-distance prefrontal-parietal connectivity (for review, see Mashour and Hudetz, 2018) so that psychotomimetic effects of ketamine (Krystal et al., 1994; Pomarol-Clotet et al., 2006) may be related to an impaired conscious access underpinned by a disruption of cerebral connectivity. Additionally, ketamine has been 
shown to disrupt top-down processes (Moran et al., 2015; van Loon et al., 2016), which are known to facilitate conscious access in healthy control subjects by enhancing cerebral activity. Interestingly, some top-down attentional amplification processes are impaired in schizophrenia (Berkovitch et al., 2018). Thus, NMDA dysfunction or impairment of attentional amplification may be necessary for the elevated consciousness threshold to induce psychotic symptoms.

A major limitation of our study is the relatively small sample size. In particular, we did not have enough power to detect significant differences in structural connectivity among the four diagnostic groups, despite finding a significant difference between patients with and without psychosis. Still, our study was built on strong theoretical hypotheses (Berkovitch et al., 2017), which considerably decreases the risk of type I errors (Oberauer and Lewandowsky, 2019).

In summary, our results demonstrate that interhemispheric and long-range posteroanterior connectivity plays a crucial role in conscious access, confirming predictions of the GNW theory of consciousness. Most importantly, we showed for the first time that deficits in conscious access may mediate impaired cerebral connectivity and psychotic symptoms, thereby providing significant anatomic counterparts to impairments observed in patients with psychosis, in addition to suggesting that psychosis and impaired conscious access may be intimately related phenomena.

\section{References}

Adams RA, Stephan KE, Brown HR, Frith CD, Friston KJ (2013) The computational anatomy of psychosis. Front Psychiatry 4:47.

Allardyce J, Suppes T, van Os J (2007) Dimensions and the psychosis phenotype. Int J Methods Psychiatr Res 16:S34-S40.

Anticevic A, Brumbaugh MS, Winkler AM, Lombardo LE, Barrett J, Corlett PR, Kober H, Gruber J, Repovs G, Cole MW, Krystal JH, Pearlson GD, Glahn DC (2013) Global prefrontal and fronto-amygdala dysconnectivity in bipolar I disorder with psychosis history. Biol Psychiatry 73:565-573.

Baron RM, Kenny DA (1986) The moderator-mediator variable distinction in social psychological research: conceptual, strategic, and statistical considerations. J Pers Soc Psychol 51:1173-1182.

Barttfeld P, Uhrig L, Sitt JD, Sigman M, Jarraya B, Dehaene S (2015) Signature of consciousness in the dynamics of resting-state brain activity. Proc Natl Acad Sci U S A 112:887-892.

Berkovitch L, Dehaene S, Gaillard R (2017) Disruption of conscious access in schizophrenia. Trends Cogn Sci 21:878-892.

Berkovitch L, Del Cul A, Maheu M, Dehaene S (2018) Impaired conscious access and abnormal attentional amplification in schizophrenia. Neuroimage Clin 18:835-848.

Boly M, Moran R, Murphy M, Boveroux P, Bruno M-A, Noirhomme Q, Ledoux D, Bonhomme V, Brichant J-F, Tononi G, Laureys S, Friston K (2012) Connectivity changes underlying spectral EEG changes during propofol-induced loss of consciousness. J Neurosci 32:7082-7090.

Butler PD, DeSanti LA, Maddox J, Harkavy-Friedman JM, Amador XF, Goetz RR, Javitt DC, Gorman JM (2003) Visual backward-masking deficits in schizophrenia: relationship to visual pathway function and symptomatology. Schizophr Res 59:199-209.

Canu E, Agosta F, Filippi M (2015) A selective review of structural connectivity abnormalities of schizophrenic patients at different stages of the disease. Schizophr Res 161:19-28.

Charles L, Gaillard R, Amado I, Krebs M-O, Bendjemaa N, Dehaene S (2017) Conscious and unconscious performance monitoring: evidence from patients with schizophrenia. Neuroimage 144:153-163.

Chkonia E, Roinishvili M, Reichard L, Wurch W, Puhlmann H, Grimsen C, Herzog MH, Brand A (2012) Patients with functional psychoses show similar visual backward masking deficits. Psychiatry Res 198:235-240.

Corlett PR, Horga G, Fletcher PC, Alderson-Day B, Schmack K, Powers AR (2019) Hallucinations and strong priors. Trends Cogn Sci 23:114-127.

Dehaene S, Changeux J-P (2011) Experimental and theoretical approaches to conscious processing. Neuron 70:200-227.
Dehaene S, Kerszberg M, Changeux J-P (1998) A neuronal model of a global workspace in effortful cognitive tasks. Proc Natl Acad Sci U S A 95:14529-14534.

Dehaene S, Artiges E, Naccache L, Martelli C, Viard A, Schürhoff F, Recasens C, Martinot MLP, Leboyer M, Martinot J-L (2003) Conscious and subliminal conflicts in normal subjects and patients with schizophrenia: the role of the anterior cingulate. Proc Natl Acad Sci U S A 100:1372213727.

Del Cul A, Dehaene S, Leboyer M (2006) Preserved subliminal processing and impaired conscious access in schizophrenia. Arch Gen Psychiatry 63:1313-1323.

Del Cul A, Baillet S, Dehaene S (2007) Brain dynamics underlying the nonlinear threshold for access to consciousness. PLoS Biol 5:e260.

Del Cul A, Dehaene S, Reyes P, Bravo E, Slachevsky A (2009) Causal role of prefrontal cortex in the threshold for access to consciousness. Brain 132:2531-2540.

Demertzi A, Tagliazucchi E, Dehaene S, Deco G, Barttfeld P, Raimondo F, Martial C, Fernández-Espejo D, Rohaut B, Voss HU, Schiff ND, Owen AM, Laureys S, Naccache L, Sitt JD (2019) Human consciousness is supported by dynamic complex patterns of brain signal coordination. Sci Adv 5:eaat7603.

Descoteaux M, Angelino E, Fitzgibbons S, Deriche R (2007) Regularized, fast, and robust analytical Q-ball imaging. Magn Reson Med 58:497-510.

Favre P, Pauling M, Stout J, Hozer F, Sarrazin S, Abé C, Alda M, Alloza C, Alonso-Lana S, Andreassen OA, Baune BT, Benedetti F, Busatto GF, Canales-Rodríguez EJ, Caseras X, Chaim-Avancini TM, Ching CRK, Dannlowski U, Deppe M, Eyler LT, et al. (2019) Widespread white matter microstructural abnormalities in bipolar disorder: evidence from megaand meta-analyses across 3033 individuals. Neuropsychopharmacology 44:2285-2293.

Fleming K, Green MF (1995) Backward masking performance during and after manic episodes. J Abnorm Psychol 104:63-68.

Fletcher PC, Frith CD (2009) Perceiving is believing: a Bayesian approach to explaining the positive symptoms of schizophrenia. Nat Rev Neurosci 10:48-58.

Forkel SJ, Thiebaut de Schotten M, Kawadler JM, Dell'Acqua F, Danek A, Catani M (2014) The anatomy of fronto-occipital connections from early blunt dissections to contemporary tractography. Cortex 56:73-84.

Goghari VM, Sponheim SR (2008) Divergent backward masking performance in schizophrenia and bipolar disorder: association with COMT. Am J Med Genet 147B:223-227.

Grandgenevre P, Vaiva G, Boloix E, Bubrovszky M, Schwan R, Laprevote V (2015) Dissociation of explicit and implicit responses during a change blindness task in schizophrenia. Neuropsychologia 71:11-17.

Green MF, Nuechterlein KH, Breitmeyer B, Mintz J (1999) Backward masking in unmedicated schizophrenic patients in psychotic remission: possible reflection of aberrant cortical oscillation. Am J Psychiatry 156:13671373.

Green MF, Lee J, Wynn JK, Mathis KI (2011) Visual masking in schizophrenia: overview and theoretical implications. Schizophr Bull 37:700-708.

Greicius MD, Supekar K, Menon V, Dougherty RF (2009) Resting-state functional connectivity reflects structural connectivity in the default mode network. Cereb Cortex 19:72-78.

Griffa A, Baumann PS, Klauser P, Mullier E, Cleusix M, Jenni R, van den Heuvel MP, Do KQ, Conus P, Hagmann P (2019) Brain connectivity alterations in early psychosis: from clinical to neuroimaging staging. Transl Psychiatry 9:62.

Guevara M, Román C, Houenou J, Duclap D, Poupon C, Mangin JF, Guevara P (2017) Reproducibility of superficial white matter tracts using diffusion-weighted imaging tractography. Neuroimage 147:703-725.

Guevara P, Duclap D, Poupon C, Marrakchi-Kacem L, Fillard P, Le Bihan D, Leboyer M, Houenou J, Mangin J-F (2012) Automatic fiber bundle segmentation in massive tractography datasets using a multi-subject bundle atlas. Neuroimage 61:1083-1099.

Hanslmayr S, Backes H, Straub S, Popov T, Langguth B, Hajak G, Bäuml KHT, Landgrebe M (2013) Enhanced resting-state oscillations in schizophrenia are associated with decreased synchronization during inattentional blindness. Hum Brain Mapp 34:2266-2275.

Henry C, Etain B (2010) New ways to classify bipolar disorders: going from categorical groups to symptom clusters or dimensions. Curr Psychiatry Rep 12:505-511. 
Herbet G, Maheu M, Costi E, Lafargue G, Duffau H (2016) Mapping neuroplastic potential in brain-damaged patients. Brain 139:829-844.

Herzog MH, Roinishvili M, Chkonia E, Brand A (2013) Schizophrenia and visual backward masking: a general deficit of target enhancement. Front Psychol 4:254.

Hesselmann G, Naccache L, Cohen L, Dehaene S (2013) Splitting of the P3 component during dual-task processing in a patient with posterior callosal section. Cortex 49:730-747.

Higashima $M$, Takeda $T$, Kikuchi $M$, Nagasawa $T$, Hirao $N$, Oka $T$, Nakamura M, Koshino Y (2007) State-dependent changes in intrahemispheric EEG coherence for patients with acute exacerbation of schizophrenia. Psychiatry Res 149:41-47.

Honey CJ, Kötter R, Breakspear M, Sporns O (2007) Network structure of cerebral cortex shapes functional connectivity on multiple time scales. Proc Natl Acad Sci U S A 104:10240-10245.

Honey CJ, Sporns O, Cammoun L, Gigandet X, Thiran JP, Meuli R, Hagmann P (2009) Predicting human resting-state functional connectivity from structural connectivity. Proc Natl Acad Sci U S A 106:20352040.

Horn A, Ostwald D, Reisert M, Blankenburg F (2014) The structural-functional connectome and the default mode network of the human brain. NeuroImage 102:142-151.

Höschel K, Irle E (2001) Emotional priming of facial affect identification in schizophrenia. Schizophr Bull 27:317-327.

Huddy VC, Aron AR, Harrison M, Barnes TRE, Robbins TW, Joyce EM (2009) Impaired conscious and preserved unconscious inhibitory processing in recent onset schizophrenia. Psychol Med 39:907-916.

Jardri R, Duverne S, Litvinova AS, Denève S (2017) Experimental evidence for circular inference in schizophrenia. Nat Commun 8:14218.

Kay SR, Fiszbein A, Opler LA (1987) The positive and negative syndrome scale (PANSS) for schizophrenia. Schizophr Bull 13:261-276.

Kelly S, Jahanshad N, Zalesky A, Kochunov P, Agartz I, Alloza C, Andreassen OA, Arango C, Banaj N, Bouix S, Bousman CA, Brouwer RM, Bruggemann J, Bustillo J, Cahn W, Calhoun V, Cannon D, Carr V, Catts S, Chen J, et al. (2018) Widespread white matter microstructural differences in schizophrenia across 4322 individuals: results from the ENIGMA Schizophrenia DTI Working Group. Mol Psychiatry 23:12611269.

King J-R, Sitt JD, Faugeras F, Rohaut B, El Karoui I, Cohen L, Naccache L, Dehaene $S$ (2013) Information sharing in the brain indexes consciousness in noncommunicative patients. Curr Biol 23:1914-1919.

Klauser P, Baker ST, Cropley VL, Bousman C, Fornito A, Cocchi L, Fullerton JM, Rasser P, Schall U, Henskens F, Michie PT, Loughland C, Catts SV, Mowry B, Weickert TW, Shannon Weickert C, Carr V, Lenroot R, Pantelis C, et al. (2017) White matter disruptions in schizophrenia are spatially widespread and topologically converge on brain network hubs. Schizophr Bull 43:425-435.

Kochunov P, Coyle TR, Rowland LM, Jahanshad N, Thompson PM, Kelly S, Du X, Sampath H, Bruce H, Chiappelli J, Ryan M, Fisseha F, Savransky A, Adhikari B, Chen S, Paciga SA, Whelan CD, Xie Z, Hyde CL, Chen X, et al. (2017) Association of white matter with core cognitive deficits in patients with schizophrenia. JAMA Psychiatry 74:958-966.

Krystal JH, Karper LP, Seibyl JP, Freeman GK, Delaney R, Bremner JD, Heninger GR, Bowers MB Jr, Charney DS (1994) Subanesthetic effects of the noncompetitive NMDA antagonist, ketamine, in humans. Psychotomimetic, perceptual, cognitive, and neuroendocrine responses. Arch Gen Psychiatry 51:199-214.

Le Bihan D, Johansen-Berg H (2012) Diffusion MRI at 25: exploring brain tissue structure and function. Neuroimage 61:324-341.

Lee U, Ku S, Noh G, Baek S, Choi B, Mashour GA (2013) Disruption of frontal-parietal communication by ketamine, propofol, and sevoflurane. Anesthesiology 118:1264-1275.

Leucht S, Samara M, Heres S, Davis JM (2016) Dose equivalents for antipsychotic drugs: the DDD method. Schizophr Bull 42 [Suppl 1]:S90-S94.

Li F, Lui S, Yao L, Ji G-J, Liao W, Sweeney JA, Gong Q (2018) Altered white matter connectivity within and between networks in antipsychotic-naive first-episode schizophrenia. Schizophr Bull 44:409-418.

Magioncalda P, Martino M, Conio B, Escelsior A, Piaggio N, Presta A, Marozzi V, Rocchi G, Anastasio L, Vassallo L, Ferri F, Huang Z, Roccatagliata L, Pardini M, Northoff G, Amore M (2015) Functional connectivity and neuronal variability of resting state activity in bipolar disorder-reduction and decoupling in anterior cortical midline structures. Hum Brain Mapp 36:666-682.

Magioncalda P, Martino M, Conio B, Piaggio N, Teodorescu R, Escelsior A, Marozzi V, Rocchi G, Roccatagliata L, Northoff G, Inglese M, Amore M (2016) Patterns of microstructural white matter abnormalities and their impact on cognitive dysfunction in the various phases of type I bipolar disorder. J Affect Disord 193:39-50.

Martino M, Magioncalda P, Huang Z, Conio B, Piaggio N, Duncan NW, Rocchi G, Escelsior A, Marozzi V, Wolff A, Inglese M, Amore M, Northoff G (2016a) Contrasting variability patterns in the default mode and sensorimotor networks balance in bipolar depression and mania. Proc Natl Acad Sci U S A 113:4824-4829.

Martino M, Magioncalda P, Saiote C, Conio B, Escelsior A, Rocchi G, Piaggio N, Marozzi V, Huang Z, Ferri F, Amore M, Inglese M, Northoff G (2016b) Abnormal functional-structural cingulum connectivity in mania: combined functional magnetic resonance imaging-diffusion tensor imaging investigation in different phases of bipolar disorder. Acta Psychiatr Scand 134:339-349.

Mashour GA, Hudetz AG (2018) Neural correlates of unconsciousness in large-scale brain networks. Trends Neurosci 41:150-160.

Mathis KI, Wynn JK, Jahshan C, Hellemann G, Darque A, Green MF (2012) An electrophysiological investigation of attentional blink in schizophrenia: separating perceptual and attentional processes. Int J Psychophysiol 86:108-113.

McClure RK (1999) Backward masking in bipolar affective disorder. Prog Neuropsychopharmacol Biol Psychiatry 23:195-206.

McIntosh AM, Maniega SM, Lymer GKS, McKirdy J, Hall J, Sussmann JED, Bastin ME, Clayden JD, Johnstone EC, Lawrie SM (2008) White matter tractography in bipolar disorder and schizophrenia. Biol Psychiatry 64:1088-1092.

Moran RJ, Jones MW, Blockeel AJ, Adams RA, Stephan KE, Friston KJ (2015) Losing control under ketamine: suppressed cortico-hippocampal drive following acute ketamine in rats. Neuropsychopharmacology 40:268-277.

Northoff G, Huang Z (2017) How do the brain's time and space mediate consciousness and its different dimensions? Temporo-spatial theory of consciousness (TTC). Neurosci Biobehav Rev 80:630-645.

Northoff G, Wainio-Theberge S, Evers K (2020) Is temporo-spatial dynamics the "common currency" of brain and mind? In quest of "spatiotemporal neuroscience”. Phys Life Rev 33:34-54.

Oberauer K, Lewandowsky S (2019) Addressing the theory crisis in psychology. Psychon Bull Rev 26:1596-1618.

Pettersson-Yeo W, Allen P, Benetti S, McGuire P, Mechelli A (2011) Dysconnectivity in schizophrenia: where are we now? Neurosci Biobehav Rev 35:1110-1124.

Pomarol-Clotet E, Honey GD, Murray GK, Corlett PR, Absalom AR, Lee M, McKenna PJ, Bullmore ET, Fletcher PC (2006) Psychological effects of ketamine in healthy volunteers. Phenomenological study. Br J Psychiatry 189:173-179.

Powers AR, Mathys C, Corlett PR (2017) Pavlovian conditioning-induced hallucinations result from overweighting of perceptual priors. Science 357:596-600.

Reuter F, Del Cul A, Audoin B, Malikova I, Naccache L, Ranjeva JP, LyonCaen O, Ali Chérif A, Cohen L, Dehaene S, Pelletier J (2007) Intact subliminal processing and delayed conscious access in multiple sclerosis. Neuropsychologia 45:2683-2691.

Reuter F, Del Cul A, Malikova I, Naccache L, Confort-Gouny S, Cohen L, Cherif AA, Cozzone PJ, Pelletier J, Ranjeva J-P, Dehaene S, Audoin B (2009) White matter damage impairs access to consciousness in multiple sclerosis. Neuroimage 44:590-599.

Román C, Guevara M, Valenzuela R, Figueroa M, Houenou J, Duclap D, Poupon C, Mangin J-F, Guevara P (2017) Clustering of whole-brain white matter short association bundles using HARDI data. Front Neuroinform 11:73.

Saccuzzo DP, Braff DL (1986) Information-processing abnormalities: traitand state-dependent components. Schizophr Bull 12:447-459.

Sarrazin S, Poupon C, Linke J, Wessa M, Phillips M, Delavest M, Versace A, Almeida J, Guevara P, Duclap D, Duchesnay E, Mangin J-F, Le Dudal K, Daban C, Hamdani N, D’Albis M-A, Leboyer M, Houenou J (2014) A multicenter tractography study of deep white matter tracts in bipolar I disorder: psychotic features and interhemispheric disconnectivity. JAMA Psychiatry 71:388-396. 
Sarubbo S, De Benedictis A, Maldonado IL, Basso G, Duffau H (2013) Frontal terminations for the inferior fronto-occipital fascicle: anatomical dissection, DTI study and functional considerations on a multi-component bundle. Brain Struct Funct 218:21-37.

Schroeder KE, Irwin ZT, Gaidica M, Nicole Bentley J, Patil PG, Mashour GA, Chestek CA (2016) Disruption of corticocortical information transfer during ketamine anesthesia in the primate brain. Neuroimage 134:459465.

Sergent C, Baillet S, Dehaene S (2005) Timing of the brain events underlying access to consciousness during the attentional blink. Nat Neurosci 8:1391-1400.

Shrout PE, Bolger N (2002) Mediation in experimental and nonexperimental studies: new procedures and recommendations. Psychol Methods 7:422445.

Skelly LR, Calhoun V, Meda SA, Kim J, Mathalon DH, Pearlson GD (2008) Diffusion tensor imaging in schizophrenia: relationship to symptoms. Schizophr Res 98:157-162.

Skudlarski P, Schretlen DJ, Thaker GK, Stevens MC, Keshavan MS, Sweeney JA, Tamminga CA, Clementz BA, O’Neil K, Pearlson GD (2013) Diffusion tensor imaging white matter endophenotypes in patients with schizophrenia or psychotic bipolar disorder and their relatives. Am J Psychiatry 170:886-898.

Souza-Queiroz J, Boisgontier J, Etain B, Poupon C, Duclap D, d'Albis M-A, Daban C, Hamdani N, Le Corvoisier P, Delavest M, Bellivier F, Guevara P, Leboyer M, Henry C, Houenou J (2016) Childhood trauma and the limbic network: a multimodal MRI study in patients with bipolar disorder and controls. J Affect Disord 200:159-164.

Sterzer P, Adams RA, Fletcher P, Frith C, Lawrie SM, Muckli L, Petrovic P, Uhlhaas P, Voss M, Corlett PR (2018) The predictive coding account of psychosis. Biol Psychiatry 84:634-643.
Sun Z, Wang F, Cui L, Breeze J, Du X, Wang X, Cong Z, Zhang H, Li B, Hong N, Zhang D (2003) Abnormal anterior cingulum in patients with schizophrenia: a diffusion tensor imaging study. Neuroreport 14:18331836.

Tingley D, Yamamoto T, Hirose K, Keele L, Imai K (2014) Mediation: R package for causal mediation analysis. J Stat Softw 59:1-38.

Tuch DS (2004) Q-ball imaging. Magn Reson Med 52:1358-1372.

Urbanski M, Thiebaut de Schotten M, Rodrigo S, Catani M, Oppenheim C, Touzé E, Chokron S, Méder J-F, Lévy R, Dubois B, Bartolomeo P (2008) Brain networks of spatial awareness: evidence from diffusion tensor imaging tractography. J Neurol Neurosurg Psychiatry 79:598-601.

van Loon AM, Fahrenfort JJ, van der Velde B, Lirk PB, Vulink NCC, Hollmann MW, Scholte HS, Lamme VAF (2016) NMDA receptor antagonist ketamine distorts object recognition by reducing feedback to early visual cortex. Cereb Cortex 26:1986-1996.

van Vugt B, Dagnino B, Vartak D, Safaai H, Panzeri S, Dehaene S, Roelfsema PR (2018) The threshold for conscious report: signal loss and response bias in visual and frontal cortex. Science 360:537-542.

Wagenmakers E-J, Lodewyckx T, Kuriyal H, Grasman R (2010) Bayesian hypothesis testing for psychologists: a tutorial on the Savage-Dickey method. Cogn Psychol 60:158-189.

Wolkin A, Choi SJ, Szilagyi S, Sanfilipo M, Rotrosen JP, Lim KO (2003) Inferior frontal white matter anisotropy and negative symptoms of schizophrenia: a diffusion tensor imaging study. Am J Psychiatry 160:572574.

Wu C-H, Hwang T-J, Chen Y-J, Hsu Y-C, Lo Y-C, Liu C-M, Hwu H-G, Liu C-C, Hsieh MH, Chien Y-L, Chen C-M, Isaac Tseng W-Y (2015) Primary and secondary alterations of white matter connectivity in schizophrenia: a study on first-episode and chronic patients using whole-brain tractography-based analysis. Schizophr Res 169:54-61. 